\title{
Simple reversible staining of proteins transferred from polyacrylamide gels onto nitrocellulose membranes
}

\author{
Hans Bloemendal *, Monique Satijn \\ Department of Biochemistry, University of Nijmegen, P.O. Box 9101, $6500 \mathrm{HB}$ Nijmegen, Netherlands
}

Received 7 July 1994

\begin{abstract}
A rapid picture of the extent of protein transfer from one- or two-dimensional polyacrylamide gels onto nitrocellulose membranes prior to immunodetection can be obtained by a rapid and reversible Amido Black staining procedure.
\end{abstract}

Keywords: Reversible protein staining; Immunoblotting

\section{Amido Black staining of proteins prior to im- munoblotting}

In the course of our studies on normal versus cataractous human lens proteins a primary goal was to construct reproducible two-dimensional crystallin maps. Crystallins are the water-soluble proteins in vertebrate eye lenses and represent about $90 \%$ of the total soluble lenticular proteins $[1,2]$.

A major difficulty encountered in such studies is the fact that crystallins in the human lens, which are primary gene products, undergo a variety of posttranslational modifications, such as deamidation, truncation, phosphorylation, glycosylation, carbamylation and oxidation, particularly upon aging [3]. These modified gene products complicate the human gel map tremendously as compared, for instance, to the corresponding map of calf lens proteins [4].

\footnotetext{
* Corresponding author.
}

In order to verify the reproducibility of the gel patterns, localization and identification of one or few proteins was carried out by immunoblotting. For a complete map this localization had to be established in respect to all other protein components that did not react with the antibody. Therefore a combination of immunoblotting and staining of the proteins was necessary. Both staining prior to and after immunoblotting were tested. Several difficulties were encountered. For instance, it appeared with Ponceau $S$ post-staining [5] that the whole blot resisted complete destaining and individual protein spots were hardly visible. Application of the sensitive colloidal gold staining [6] had the same negative result. The blot kept a violet background. Therefore we searched for a reversible pre-staining method which allows the application of preincubation with a protein like bovine serum albumin (BSA), a blocking agent that prevents background staining in immunoblotting. Pre-staining with Ponceau $S$ failed due to the low amount of lens proteins required for immunoblotting 


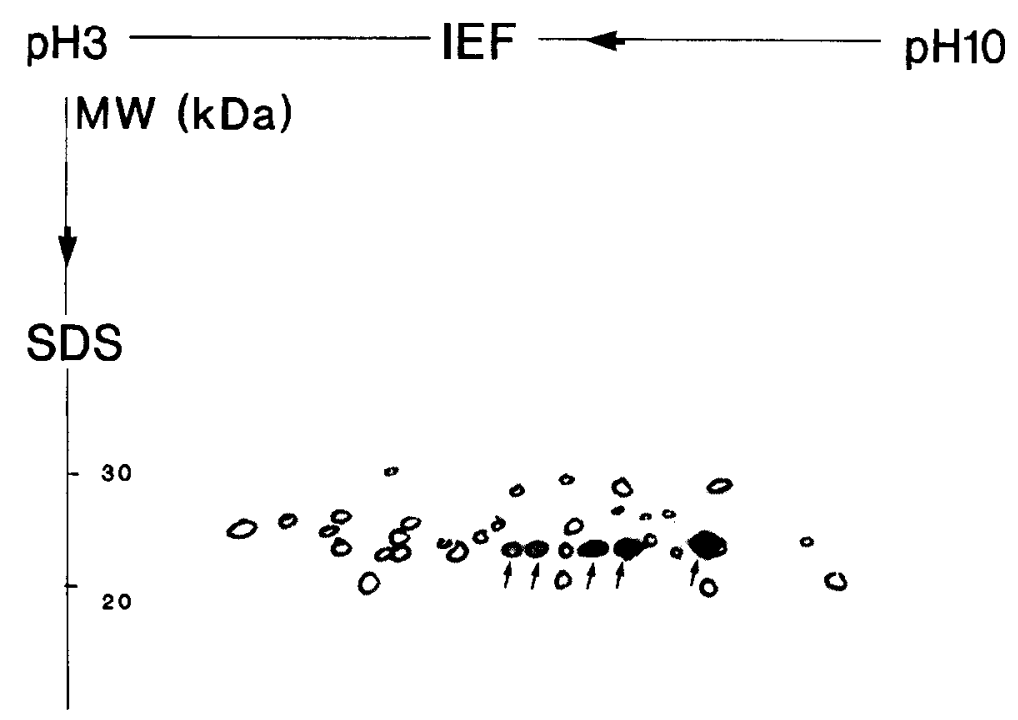

Fig. 1. Two-dimensional immunoblot of human water-soluble lens proteins, prestained with Amido Black. Immunodetection with anti- $\alpha$ B-crystallin (arrows), other crystallins marked with indelible ink.

(20 $\mu \mathrm{g}$ of protein to be separated into about 150 spots with a variation of 5-300 ng per individual spot). Gold staining gave unsatisfactory results too in that, only a spotty pattern was obtained. Only Amido Black staining appeared to be a reversible and satisfactory method (compare Table 1). Previously, Syu and Kahan [7] described a staining procedure for nitrocellulose membranes with Amido Black. However, their method differs in that the membranes were blocked with gelatin before immunoblotting. Moreover blue background protein spots not reacting with the antibodies remain on the membrane next to those which have reacted and appeared in purple. This can lead to erroneous evaluation if the immuno-positive spots are faint and hard to distin- guish from the blue-stained non immuno-reactive spots.

\section{The protocol}

(1) Proteins are transferred from the 2D-gels onto nitrocellulose membranes according to standard methods for electroblotting.

(2) The blot is immersed in an Amido Black solution (2.5 g Amido Schwarz (Merck, Darmstadt); $250 \mathrm{ml}$ methanol; $215 \mathrm{ml}$ deionized, distilled water; $35 \mathrm{ml}$ acetic acid) while shaking for 3-6 $\mathrm{s}$, followed by immediate removal of the stain.

Table 1

Advantages and disadvantages of different staining methods for proteins on nitrocellulose membranes

\begin{tabular}{lll}
\hline Staining method & Advantage & Disadvantage \\
\hline Ponceau S & Rapid, cheap, reversible & Not sensitive enough \\
Colloidal Gold & Sensitive & Slow, expensive, spotty pattern \\
Amido Black & Sensitive, rapid, cheap, reversible & \\
\hline
\end{tabular}


Longer contact with the stain would interfere with the destaining procedure.

(3) Destaining is carried out for 3-5 min by rinsing with running deionized water. The spots then become visible against a faint blue background.

(4) The localization of the spots can be marked either by photography or careful encircling with indelible ink without damaging the membrane (see Fig. 1).

(5) Thereafter the blot is further destained by shaking for another $30 \mathrm{~min}$ in deionized water, followed by incubation in 1\% BSA in PBS/Tween for $1 \mathrm{~h}$. This step is required for immuno-detection.

All remaining stain, including that coupled to proteins, vanishes and the antibody can react with its corresponding antigen. The background appears virtually white.

\section{Conclusion}

This extremely simple method, which appeared to be not damaging to nitrocellulose blots in approxi- mately hundred experiments, gives a very rapid picture of the extent of protein transfer from the gel and allows correlation of the mutual positions of proteins with those detected with the aid of antibodies.

\section{Acknowledgements}

This work has been supported by an Alcon grant.

\section{References}

[1] H. Bloemendal, Science, 197 (1977) 127.

[2] H. Bloemendal, Crit. Rev. Biochem., 12 (1982) 1.

[3] P.J.T.A. Groenen, K.B. Merck, W.W. de Jong and H. Bloemendal, Eur. J. Biochem., 225 (1994) 1.

[4] G.A.M. Berbers, W.A. Hoekman, H. Bloemendal, W.W. de Jong, T. Kleinschmidt and G. Braunitzer, Eur. J. Biochem., 139 (1984) 467.

[5] O. Salinovich and R.C. Montelaro, Anal. Biochem., 156 (1986) 341.

[6] M. Moeremans, G. Daneels and J. de Mey, Anal. Biochem., 145 (1985) 315.

[7] W-Ir. Syu and L. Kahan, J. Immunol. Methods, 103 (1987) 247. 